\title{
Einzelzellkultivierung
}

\section{Ein Wechselbad für Mikroben: Wachsen unter dynamischen Umweltbedingungen}

\author{
LUISA BLÖBAUM, SARAH TÄUBER, ALEXANDER GRÜNBERGER \\ MULTISCALE BIOENGINEERING UND CENTRUM FÜR BIOTECHNOLOGIE (CEBITEC), \\ UNIVERSITÄT BIELEFELD
}

Microfluidic technologies are increasingly used within microbiology for screening and improved understanding of cellular behaviour. The presented system for dynamic microfluidic single-cell cultivation - dMSCC - offers a new cultivation method for microbial microcolonies at dynamic environments with oscillation between seconds to hours. This tool has a high potential to improve the understanding of cellular behaviour at dynamic environments as omnipresent in nature and lab-scale cultivation systems.

DOI: $10.1007 / \mathrm{s} 12268-021-1626-0$

(C) Die Autorinnen und Autoren 2021
Im natürlichen und im künstlichen Lebensraum, dem Bioreaktor, sind Bakterien sich ändernden Umweltbedingungen ausgesetzt [1, 2]. Die Umgebung beeinflusst die Regulations- und Genexpressionsebene von Bakterien und steuert dadurch das Verhalten einzelner Zellen bis hin zu ganzen Popula- tionen [3]. Um das Verhalten von Bakterien unter diesen Bedingungen untersuchen $\mathrm{zu}$ können, müssen Umweltbedingungen bei Kultivierungen definiert, präzise kontrolliert und geändert werden können. In traditionellen Kultivierungsgefäßen, wie dem Schüttelkolben, ist dies nur bedingt möglich. Daher
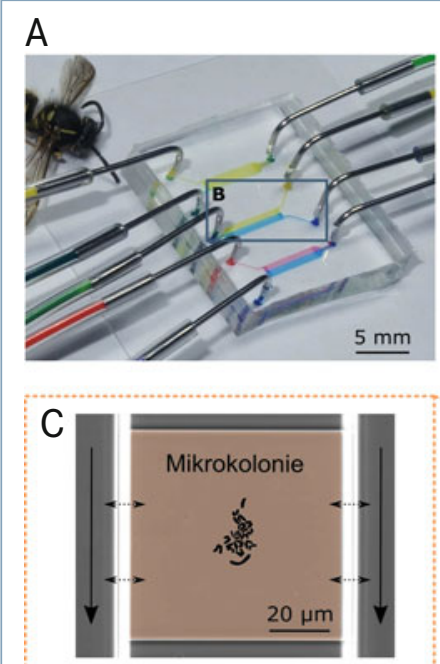

$\leftrightarrow$ Diffusion $\longrightarrow$ Laminarer Fluss

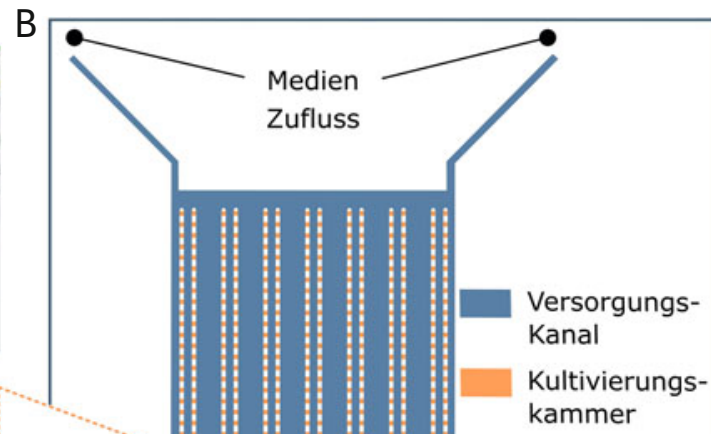

werden neue Kultivierungs- und Untersuchungsmethoden benötigt. Mikrofluidische Einzelzellkultivierung zeichnet sich hier als ein wertvolles Werkzeug ab, um Zellen bei definierter Umweltveränderung und mit voller Einzelzellauflösung kultivieren zu können.

Individuen im Fokus: mikrofluidische Einzelzellkultivierung

Mit Mikrofluidik können sehr kleine, definierte Flüssigkeitsmengen präzise gesteuert werden. Es ist möglich, einzelne Bakterien oder Mikrokolonien in Polydimethylsiloxan(PDMS)-basierten Chips (Abb. 1A) zu kultivieren und zu analysieren. Kultivierungskammern mit einer Höhe von etwa $1 \mu \mathrm{m}$ sind auf dem Chip in mehreren Reihen angeordnet, sodass das Wachstum vieler Mikrokolonien in einer Ebene gleichzeitig beobachtet werden kann (Abb. 1B). Einzelne Zellen werden in diesen Kammern gefangen, indem sie zwischen PDMS und Objektträger eingeklemmt werden (Abb. 1C). Dort können sie sich lediglich durch Teilung und Verdrängung fortbewegen und werden über Diffusion aus dem Versorgungskanal mit Nährstoffen versorgt (Abb. 1C). Aufgrund der geringen Diffusionsstrecke sind die Nährstoffe innerhalb weniger Sekunden [4] ausgetauscht, sodass in Kammer und Kanal die gleichen Bedingungen herrschen. Durch live-cell imaging können nun Zellzahl, Wachstumsverhalten und Morphologie sowie die Verwandtschaftsverhältnisse und individuelle Vergangenheit einer einzelnen Zelle analysiert werden. Die hohe Parallelisierung der Kammern ermöglich die Analyse einer statistisch relevanten Menge an Zellen.

Dynamische Umweltbedingungen: näher an der Realität

Die Umwelt zu ändern, bedeutet in der mikrofluidischen Einzelzellkultivierung, die gleiche Kammer mit einem anderen Medium zu versorgen. Um den Medienfluss in unserem System dynamic microfluidic single-cell cultivation (dMSCC) zu steuern, verwenden wir Druckunterschiede zwischen den Medienreservoirs. Die Medien werden über 

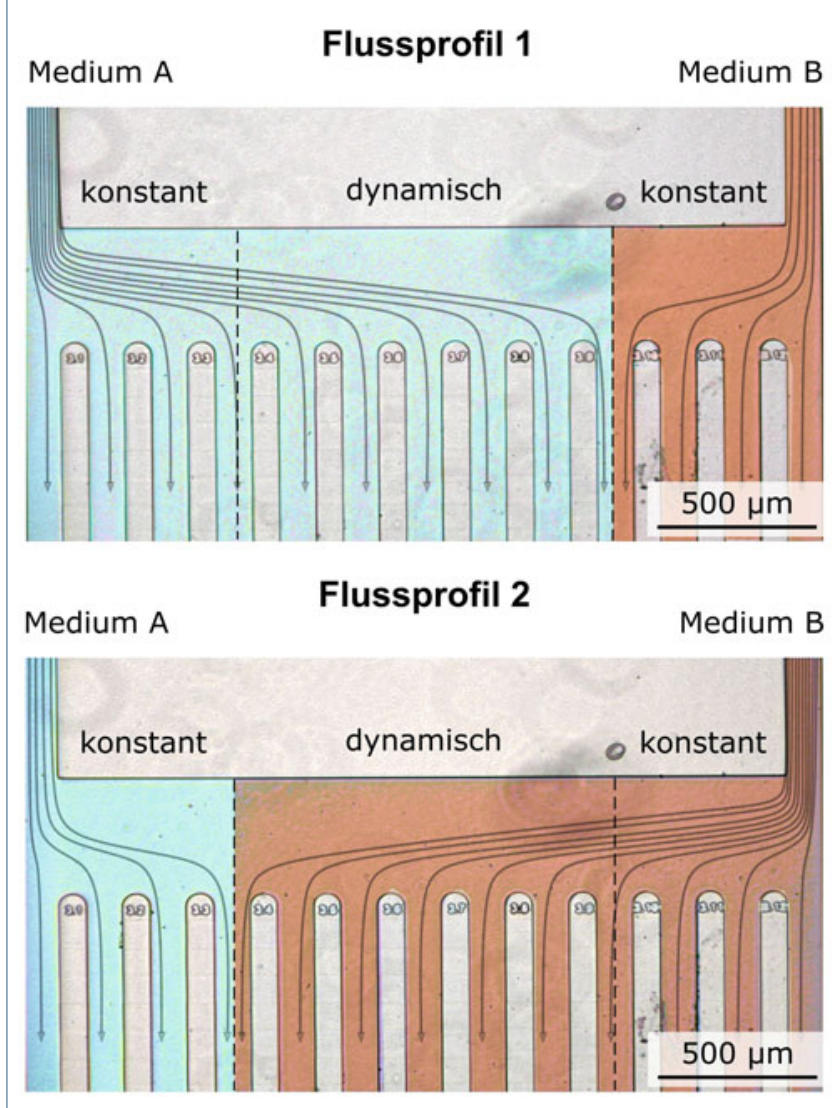

4 Abb. 2: Wechselprinzip der dMSCC: Über Druckunterschiede zwischen zwei Flüssigkeitsströmen, hier Medium A und B, kann die laminare Grenzschicht verschoben und verschiedene Flussprofile ausgebildet werden. Kammern im dynamischen Bereich erfahren Umweltschwankungen, während die Umwelt der Kontrollbereiche konstant bleibt. Modifiziert aus [4].

mer diffusiv innerhalb von etwas weniger als $5 \mathrm{~s}$ ausgetauscht wird [4]. Neben schnellen Wechseln zwischen zwei Bedingungen ist so auch die definierte Versorgung der Mikrokolonie in der Kammer gewährleistet.

\section{Fallbeispiel: erzwungenes Intervallfasten für Corynebacterium}

Das Konzept dMSCC ist auf eine Vielzahl von Fragestellungen anwendbar. Eine Kultivierung mit einem simplen Wechsel,

zwei Zuflüsse in den Chip geleitet (Abb. 1B). Aufgrund physikalischer Effekte ist der Fluss in der Mikrofluidik laminar, sodass Vermischung zwischen den Medien nur über Diffusion stattfindet. Über die Druckunterschiede kann die laminare Grenzschicht zwischen den beiden Medien verschoben werden (Abb. 2), woraufhin das Medium in der Kam- einem Stresspuls oder Oszillationen von Medien können mit dem gleichen Chipdesign durchgeführt werden.

Als Fallbeispiel untersuchten wir die Auswirkungen von Oszillationen zwischen Komplexmedium - BHI-Medium - und nähstofffreien Medium - PBS-Puffer - auf das Wachstumsverhalten von Mikrokolonien von Corynebacterium glutamicum [4]. Über den Gesamtverlauf der Kultivierung blieben die Zellen immer die Hälfte der Zeit in Medium oder Puffer. Dieses Verhältnis behielten wir in unserer Studie bei, während wir die Intervalldauer von Medium und Puffer im Stunden- bis Sekundenbereich variierten. Eine Intervalldauer von 45 min führt beispielsweise zu einem Wachstumsstopp in den Hungerzeiten (Abb. 3A), sodass die Wachstumskurve Stufen zeigt (Abb. 3B, graue Kurve). Über den Kultivierungszeitraum verringern die Stufen so die durchschnittliche Wachstumsgeschwindigkeit der Kolonie $\left(\mu_{\text {Kolonie }}\right)$. Bei einer Intervalldauer zwischen 5 und 15 min bricht $\mu_{\text {Kolonie }}$ wesentlich stärker ein. Bei Intervalldauern im Sekundenbereich (Abb. 3C, oranger Bereich) erholt sich $\mu_{\text {Kolonie }}$ wieder, erreicht jedoch nie die maximale Wachstumsgeschwindigkeit von $0,92 \mathrm{~h}^{-1}$ der BHI-Kontrolle (Abb. 3B, grüne Kurve). Wir vermuten, dass das veränderte Verhalten sich darauf zurückführen lässt, wie sehr und zu welchem Zeitpunkt die Oszillationen in zellulären Regulationsmechanismen eingreifen [5]. Wir führen gegenwärtig detailliertere Studien durch, um dieses Phänomen im Ansatz besser verstehen und erklären zu können.

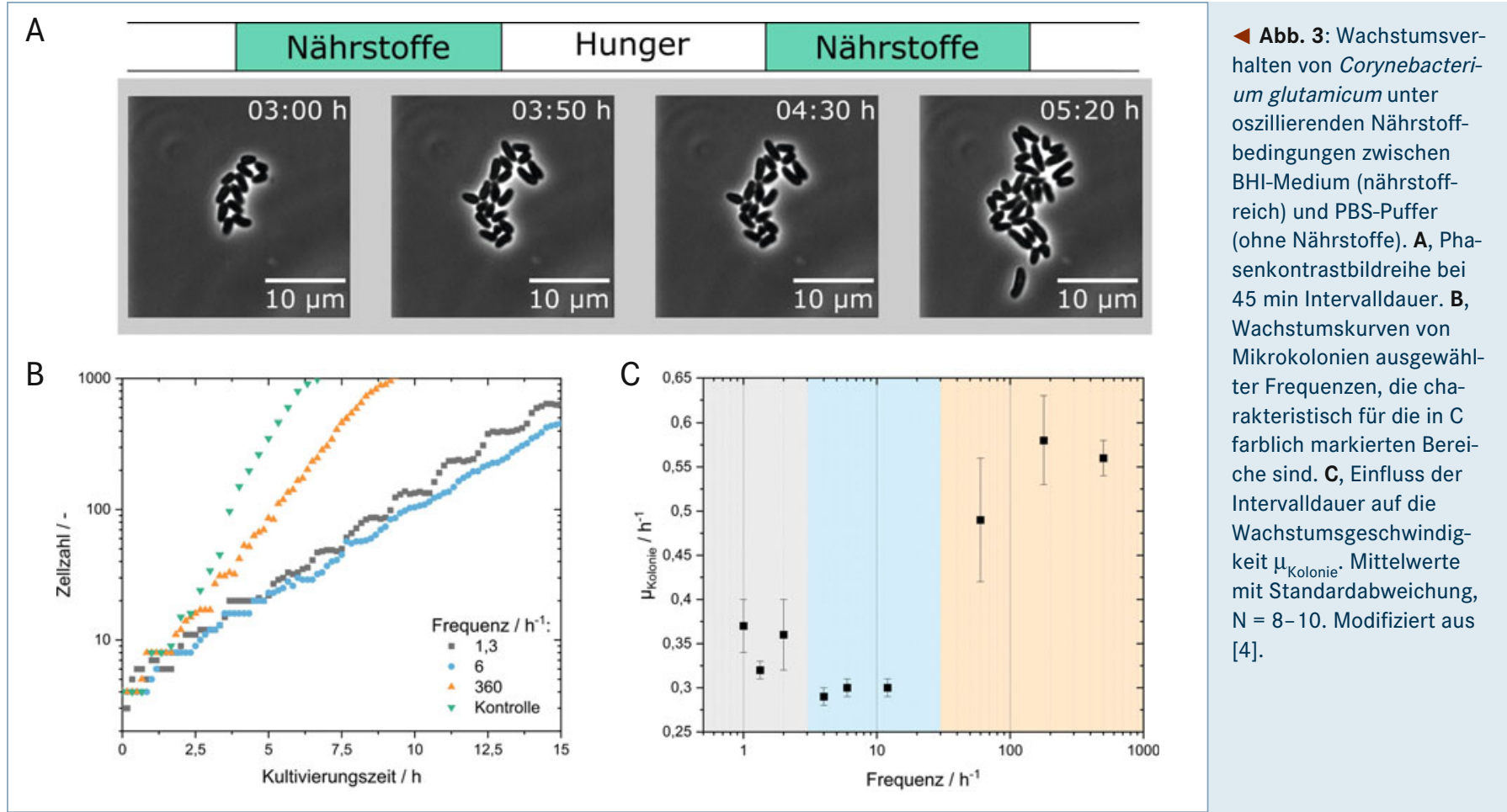




\section{Dynamische Einzelzellkultivierung: Blick in die Zukunft}

Die dynamische Einzelzellkultivierung dMSCC hat sich als ein Werkzeug mit hohem Potenzial für die Untersuchung von Umweltschwankungen auf Bakterien erwiesen. Die Etablierung der Technik in mikrobiologischen Laboren steht jedoch noch am Anfang und benötigt Verbesserungen und Weiterentwicklungen. Zeitgleiche Wechsel zwischen mehreren Parametern, aber auch die Möglichkeit, die Begasung dynamisch zu ändern, werden dMSCC weiter voranbringen. Gleichzeitig limitiert die Bildgebung als zentrales Analysewerkzeug für Kultivierung unser System, da die Daten nur optisch erhoben werden können und gegenwärtig noch auf Wachstum beschränkt sind. Die Verwendung von fluoreszierenden Biosensoren ist daher ein wichtiger Baustein, um Prozesse im Inneren der Zelle sichtbar zu machen [6]. Auch andere optische Verfahren, wie die RamanSpektroskopie, bieten in Kombination mit Einzelzellkultivierung eine Zukunftsperspektive, um metabolische Dynamiken in Echtzeit zu analysieren [7].

Dynamische Umweltbedingungen sind nicht nur für Grundlagenforschung in der Mikrobiologie interessant. In industriellen Bioprozessen sind Mikroben Schwankungen von Nährstoffen, pH-Werten und Gelöstsauerstoff im Sekunden- bis Minutenbereich ausgesetzt $[2,8]$. Doch wie beeinflussen diese die Produktivität und Heterogenität einer Kultur im Bioprozess [9]? Mit dMSCC sind einige dieser Schwankungen bereits technisch realisierbar, doch die Kombination von Parameterschwankungen, um einen Bioprozess abzubilden, bleibt eine Herausforderung.

\section{Literatur}

[1] Shade A, Jones SE, McMahon KD (2008) The influence of habitat heterogeneity on freshwater bacterial community composition and dynamics. Environ Microbiol 10: 10571067

[2] Lara AR, Galindo E, Ramírez OT et al. (2006) Living with heterogeneities in bioreactors: Understanding the effects of environmental gradients on cells. MB 34:355382

[3] Rosenzweig RF, Adams J (1994) Microbial adaptation to a changeable environment: Cell-cell interactions mediate physiological and genetic differentiation. Bioessays 16 715-717

[4] Täuber S, Golze C, Ho P et al. (2020) dMSCC: A microfluidic platform for microbial single-cell cultivation of Corynebacterium glutamicum under dynamic environmental medium conditions. Lab Chip 20: 4442-4455 tal medium conditions. Lab Chip 20: 4442-4455
[5] Bervoets I, Charlier D (2019) Diversity, versatility and complexity of bacterial gene regulation mechanisms: Opportunities and drawbacks for applications in synthetic biology. FEMS Microbiol Rev 43: 304-339

[6] Dusny C, Grünberger A (2020) Microfluidic single-cell analysis in biotechnology: From monitoring towards understanding. Curr Opin Biotechnol 63: 26-33
[7] Tao Z, Zhang P, Oin Z et al. (2016) Poly(3-hydroxybutyrate) anabolism in Cupriavidus necator cultivated at various carbon-to-nitrogen ratios: Insights from single-cell

Raman spectroscopy. J Biomed Opt 21: 97005

[8] Enfors S-0, Jahic M, Rozkov A et al. (2001) Physiological responses to mixing in large scale bioreactors. J Biotechnol 85: 175-185

[9] Heins A-L, Weuster-Botz D (2018) Population heterogeneity in microbial bioprocesses: Origin, analysis, mechanisms, and future perspectives. Bioprocess Biosyst Eng 41: $889-916$

Funding note: Open Access funding enabled and organized by Projekt DEAL. Open Access: Dieser Artikel wird unter der Creative Commons

Verne die Nutzun Vervielfaltigung, Bearbeitung, Verbreitung und Wiedergabe in jeglichem und die Lizenz beifügen und angeben, ob Änderungen vorgenommen wurden. Die in diesem Artikel enthaltenen Bilder und sonstiges Drittmaterial unterliegen (liesem Artikel enthaltenen Bilder und sonstiges Drittmaterial unterliegen Abbildungslegende nichts anderes ergibt. Sofern das betreffende Material nicht unter der genannten Creative Commons Lizenz steht und die betreffende Handlung nicht nach gesetzlichen Vorschriften erlaubt ist, ist für die oben aufgeführten Weiterverwendungen des Materials die Einwilligung des jeweiligen Rechteinhabers einzuholen. Weitere Details zur Lizenz entnehmen Sie bitte der Lizenzinformation auf http://creativecommons.org/
licenses/by/4.0/deed.de.

\section{Korrespondenzadresse:}

Prof. Dr.-Ing. Alexander Grünberger

Multiscale Bioenginering

Technische Fakultät

Universität Bielefeld

Universitätsstraße 25

D-33615 Bielefeld

alexander.gruenberger@uni-bielefeld.de

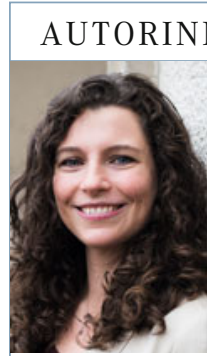

Hier steht eine Anzeige.

Luisa Blöbaum

2014-2020 Studium molekulare Biotechnologie an der Universität Bielefeld. Seit 2021 wissenschaftliche Mitarbeiterin mit dem Ziel Promotion in der AG „Multiscale Bioengineering " an der Technischen Fakultät der Universität Bielefeld.

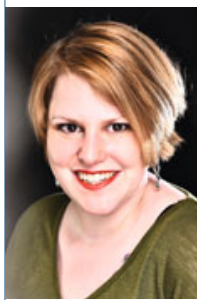

Sarah Täuber

2013-2018 Physik- und Biophysikstudium an der Universität Bielefeld. Seit 2018 wissenschaftliche Mitarbeiterin mit dem Ziel der Promotion an der Technischen Fakultät der Universität Bielefeld in der AG „Multiscale Bioengineering“.

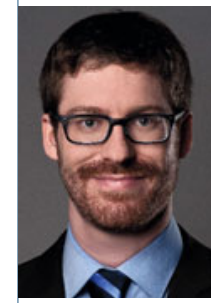

Alexander Grünberger 2004-2010 Studium Bioingenieurswesen am KIT. 2010-2015 Promotion am Institut Bio- und Geowissenschaften (IBG-1) des Forschungszentrums Jülich $\mathrm{GmbH}$ in der AG „Microscale Bioengineering " und der RWTH Aachen. 2015-2017 Postdoktorand am IBG1. 2017-2021 W1-Professor und seit 2021 W2-Professor der AG „Multiscale

Bioengineering " an der Technischen Fakultät der Universität Bielefeld. 